News and Views

\section{MALDI-TOF mass spectrometry in clinical diagnostic microbiology}

Microbial identification in clinical diagnostic laboratories mainly relies on conventional phenotypic and gene sequencing identification techniques. Recently development of matrix-assisted laser desorption ionization time-of-flight mass spectrometry (MALDI-TOF MS) devices has revolutionized the routine identification of microorganisms in clinical microbiology laboratories. This is an easy, rapid, high throughput, low-cost, and efficient identification technique and has been adapted to the constraint of clinical diagnostic laboratories. This technology has the potential to replace and/or complement conventional identification techniques for both bacterial and fungal strains.

The resolution of MALDI-TOF MS allows accurate identification at the species level of most Grampositive and Gram-negative bacterial strains, with the exception of a few difficult strains that require more attention and further development of the method, provided standardized procedures are used. Similarly, the routine identification by MALDI-TOF MS of yeast isolates is reliable and much quicker than conventional techniques. Recent studies have shown that MALDI-TOF MS has also the potential to accurately identify filamentous fungi and dermatophytes.

Numerous studies on different systems available demonstrate the reliability and accuracy of the method, and new frontiers have been explored besides microbial species level identification, such as direct identification of pathogens from positive blood cultures and drug susceptibility detection. Moreover, MALDI-TOF MS has been used successfully for microbial typing and identification at the subspecies level, demonstrating that this technology is a potential efficient tool for epidemiological studies and for taxonomical classification

Source: FEMS Microbiol Rev. 2012 Mar;36(2):380-407. 